\title{
Universiteit
}

Leiden

The Netherlands

\section{Cloning and sequencing of the tuf genes of Streptomyces coelicolor} A3(2)

Wezel, G.P. van; Woudt, L.P.; Vervenne, R.; Verdurmen, M.L.A.; Vijgenboom, E.; Bosch, L.

\section{Citation}

Wezel, G. P. van, Woudt, L. P., Vervenne, R., Verdurmen, M. L. A., Vijgenboom, E., \& Bosch, L. (1994). Cloning and sequencing of the tuf genes of Streptomyces coelicolor A3(2). Bba Gene Structure And Expression, 1219(2), 543-547. doi:10.1016/0167-4781(94)90085-X

Version: $\quad$ Publisher's Version

License: $\quad$ Licensed under Article 25fa Copyright Act/Law (Amendment Taverne)

Downloaded from: https://hdl.handle.net/1887/3239410

Note: To cite this publication please use the final published version (if applicable). 


\title{
Cloning and sequencing of the tuf genes of Streptomyces coelicolor A3(2)
}

\author{
Gilles P. van Wezel *, Lambertus P. Woudt ${ }^{1}$, Richard Vervenne, Marlou L.A. Verdurmen ${ }^{2}$, \\ Erik Vijgenboom, Leendert Bosch \\ Leiden University, Gorlaeus Laboratories, P.O. Box 9502, 2300 RA Leiden, The Netherlands
}

Received 27 April 1994

\begin{abstract}
Two tuf genes are present in Streptomyces coelicolor A3(2), which have been cloned and sequenced. These genes show a high degree of nucleotide sequence identity to the tuf1 and tuf3 genes of Streptomyces ramocissimus: the tufl genes are $94 \%$ identical, the tuf3 genes $87 \%$. S. coelicolor tuf1 encodes a protein of 396 amino acids, while tuf3 encodes a protein of 391 amino acids.
\end{abstract}

Keywords: Elongation factor Tu; Sequence comparison; Gene cloning; (Streptomyces)

One of the most abundant proteins in the bacterial cytoplasm is the tuf-encoded polypeptide chain elongation factor Tu (EF-Tu). Two tuf genes occur in Escherichia coli, encoding elongation factors EF-TuA and EF-TuB which differ only in their C-terminal amino acid $[1,2]$. The proteins occur in the bacterial cell in a $1: 1$ ratio [3], and are functionally indistinguishable.

Recently, we showed that in the kirromycin producer Streptomyces ramocissimus three tuf genes occur, which have been cloned and sequenced [4]. Analysis of these genes revealed that they are unexpectedly heterogeneous: tuf1 and tuf2 show $85 \%$ nucleotide sequence identity, whereas tuf 3 shows only $70 \%$ identity to tuf1 and tuf2, which is in striking contrast to the much higher similarities found among tuf genes in other microorganisms [5,6]. EF-Tu1 has been shown to be a genuine elongation factor, but no physiological function could be revealed for the other two putative elongation factors. Hybridization analysis of other streptomycetes has shown that Streptomyces coelicolor

\footnotetext{
* Corresponding author. Fax: + 3171274340 .

E-mail: wezel_g@chem.leidenuniv.nl.

${ }^{1}$ Present address: Zaadunie BV, Department of Biotechnology, P.O. Box 26, 1600 AA Enkhuizen, The Netherlands.

${ }^{2}$ Present address: Centocor Europe BV, P.O. Box 251, 2300 AG Leiden, The Netherlands.
}

and Streptomyces lividans, genetically the best-characterised streptomycetes, have only two tuf genes.

Cloning of the S. coelicolor tuf1 and tuf3 genes. $S$. coelicolor presumably has two tuf genes, designated tufl and tuf3 by analogy to their homologues in $S$. ramocissimus. From Southern hybridization data it was concluded that tufl could be cloned as an approx. 4.5 $\mathrm{kb}$ BamHI fragment and tuf3 as an approx. $10 \mathrm{~kb}$ Bam HI fragment [7]. S. coelicolor M145 total DNA was digested with Bam HI and separated electrophoretically on a $0.7 \%$ agarose gel in TAE buffer, whereupon fragments of the appropriate size were isolated from the gel.

For tuf1, these fragments were subcloned into Bam HI-digested pAT153 [8] and screened by hybridization of the $E$. coli colonies with the $244 \mathrm{bp}$ SmaI fragment internal to the $S$. ramocissimus tuf1 gene, which encodes most of the GTP-binding region. One positive signal was obtained, which appeared to contain a $4.3 \mathrm{~kb}$ insert, corresponding to the size expected on the basis of Southern hybridization data. This clone, which was designated pASCT1-1, contained the gene homologous to $S$. ramocissimus tuf1, as shown below.

For cloning of tuf3, fragments were cloned into pBR329 [9] and plasmid DNA was isolated from 600 colonies in pools of 24 . The DNA was digested with 
$B a m \mathrm{HI}$, subjected to agarose gel electrophoresis and blotted onto a Hybond-N nylon filter. Pools with the correct insert were identified on the basis of hybridization signals obtained with the $600 \mathrm{bp}$ Sal I fragment from $S$. ramocissimus tuf3. After repeated colony purification and screening, one DNA preparation yielding an unambiguous positive signal was obtained. Bam $\mathrm{HI}$ digestion proved the DNA to contain a $10.3 \mathrm{~kb}$ insert. The clone was designated pBSCT3-1. It was shown to contain the tuf 3 gene, as is demonstrated below.

Sequences of the inserts of pASCT1-1 and pBSCT3-1: nucleotide and amino acid comparisons. Sequencing of

1 CTCGAGCCGATGATGGCCGTCGAGGTCACCACGCCCGAGGACTACATGGGCGACGTCATCGGCGACATCAACTCC $\begin{array}{lllllllllllllllllllllllll}\mathrm{L} & \mathrm{E} & \mathrm{P} & \mathrm{M} & \mathrm{M} & \mathrm{A} & \mathrm{V} & \mathrm{E} & \mathrm{V} & \mathrm{T} & \mathrm{T} & \mathrm{P} & \mathrm{E} & \mathrm{D} & \mathrm{Y} & \mathrm{M} & \mathrm{G} & \mathrm{D} & \mathrm{V} & \mathrm{I} & \mathrm{G} & \mathrm{D} & \mathrm{I} & \mathrm{N} & \mathrm{S}\end{array}$ CGCCGTGGCCAGATCCAGGCCATGGAGGAGCGGATGGGTGCCCGCGTCGTGAAGGGCCTCGTGCCGCTGTCGGAG $\begin{array}{llllllllllllllllllllllllll}R & R & G & Q & I & Q & A & M & E & E & R & M & G & A & R & V & V & K & G & L & V & P & L & S & E\end{array}$ ATGTTCGGCTACGTCGGAGACCTCCGCAGCAAGACGTCGGGTCGCGCAAGCTACTCGATGCAGTTCGACTCCTAC $\begin{array}{lllllllllllllllllllllllll}M & F & G & Y & V & G & D & L & R & S & K & T & S & G & R & A & S & Y & S & M & Q & F & D & S & Y\end{array}$ GCCGAGGTTCCCCGGAACGTCGCCGAGGAGATCATCGCGAAGGCCAAGGGCGAGTAACGGGCTACTCCGTTTAAC $\begin{array}{llllllllllllllllll}A & E & V & P & R & N & V & A & E & E & I & I & A & K & A & K & G & E\end{array}$ GGACCCCGTTCTCACGCTTTAGGCTTGACCCCGGAGCCTGCATGGGGCATTCCGCCGTGAACCCGGTGGAATGCC CCCGGCACCCGGGCTTTCCAGCAAAGATCACCTGGCGCCGATGAGTAAGGCGTACAGAACCACTCCACAGGAGGA CCCCAGTGGCGAAGGCGAAGTTCGAGCGGACTAAGCCGCACGTCAACATCGGCACCATCGGTCACATCGACCACG $\begin{array}{lllllllllllllllllllllllll}V & A & K & A & K & F & E & R & T & K & P & H & V & N & I & G & T & I & G & H & I & D & H & G\end{array}$ GTAAGACGACCCTCACGGCCGCCATTACCAAGGTGCTGCACGACGCGTACCCGGACATCAACGAGGCGTCGGCGT $\begin{array}{llllllllllllllllllllllllllllllllll}K & \mathrm{~T} & \mathrm{~T} & \mathrm{~L} & \mathrm{~T} & \mathrm{~A} & \mathrm{~A} & \mathrm{I} & \mathrm{T} & \mathrm{K} & \mathrm{V} & \mathrm{L} & \mathrm{H} & \mathrm{D} & \mathrm{A} & \mathrm{Y} & \mathrm{P} & \mathrm{D} & \mathrm{I} & \mathrm{N} & \mathrm{E} & \mathrm{A} & \mathrm{S} & \mathrm{A} & \mathrm{F}\end{array}$ TCGACCAGATCGACAAGGCTCCCGAAGAGCGCCAGCGCGGTATCACCATCTCGATCGCGCACGTCGAGTACCAGA $\begin{array}{lllllllllllllllllllllllll}\mathrm{D} & \mathrm{Q} & \mathrm{I} & \mathrm{D} & \mathrm{K} & \mathrm{A} & \mathrm{P} & \mathrm{E} & \mathrm{E} & \mathrm{R} & \mathrm{Q} & \mathrm{R} & \mathrm{G} & \mathrm{I} & \mathrm{T} & \mathrm{I} & \mathrm{S} & \mathrm{I} & \mathrm{A} & \mathrm{H} & \mathrm{V} & \mathrm{E} & \mathrm{Y} & \mathrm{Q} & \mathrm{T}\end{array}$ CCGAGGCGCGTCACTACGCCCACGTCGACTGCCCCGGTCACGCCGACTACATCAAGAACATGATCACGGGTGCCG $\begin{array}{llllllllllllllllllllllllll}\mathrm{E} & \mathrm{A} & \mathrm{R} & \mathrm{H} & \mathrm{Y} & \mathrm{A} & \mathrm{H} & \mathrm{V} & \mathrm{D} & \mathrm{C} & \mathrm{P} & \mathrm{G} & \mathrm{H} & \mathrm{A} & \mathrm{D} & \mathrm{Y} & \mathrm{I} & \mathrm{K} & \mathrm{N} & \mathrm{M} & \mathrm{I} & \mathrm{T} & \mathrm{G} & \mathrm{A} & \mathrm{A}\end{array}$

CGCAGATGGACGGCGCCATCCTCGTGGTCGCCGCCACCGACGGCCCGATGCCGCAGACCAAGGAGCACGTGCTCC $\begin{array}{llllllllllllllllllllllllll}\mathrm{Q} & \mathrm{M} & \mathrm{D} & \mathrm{G} & \mathrm{A} & \mathrm{I} & \mathrm{L} & \mathrm{V} & \mathrm{V} & \mathrm{A} & \mathrm{A} & \mathrm{T} & \mathrm{D} & \mathrm{G} & \mathrm{P} & \mathrm{M} & \mathrm{P} & \mathrm{Q} & \mathrm{T} & \mathrm{K} & \mathrm{E} & \mathrm{H} & \mathrm{V} & \mathrm{L} & \mathrm{L}\end{array}$

TGGCCCGCCAGGTCGGCGTTCCGTACATCGTGGTCGCCCTGAACAAGGCCGACATGGTGGACGACGAGGAGATCC $\begin{array}{lllllllllllllllllllllllll}A & R & Q & V & G & V & P & Y & I & V & V & A & L & N & K & A & D & M & V & D & D & E & E & I & L\end{array}$

TGGAGCTCGTCGAGCTCGAGGTGCGTGAGCTCCTCTCCGAGTACGAGTTCCCGGGCGACGACGTTCCCGTCGTCA $\begin{array}{llllllllllllllllllllllllll}E & \mathrm{~L} & \mathrm{~V} & \mathrm{E} & \mathrm{L} & \mathrm{E} & \mathrm{V} & \mathrm{R} & \mathrm{E} & \mathrm{L} & \mathrm{L} & \mathrm{S} & \mathrm{E} & \mathrm{Y} & \mathrm{E} & \mathrm{F} & \mathrm{P} & \mathrm{G} & \mathrm{D} & \mathrm{D} & \mathrm{V} & \mathrm{P} & \mathrm{V} & \mathrm{V} & \mathrm{K}\end{array}$

GTCGCGGTACGGTCGTCACCGGCCGCATCGAGCGTGGTGTCCTCAAGGTCAACGAGACCGTCGACATCATCG

1201 GCATCAAGACCGAGAAGACCACCACCACGGTCACCGGCATCGAGATGTTCCGCAAGCTCCTCGACGAGGGCCAGG $\begin{array}{lllllllllllllllllllllllll}\mathrm{I} & K & \mathrm{~T} & \mathrm{E} & \mathrm{K} & \mathrm{T} & \mathrm{T} & \mathrm{T} & \mathrm{T} & \mathrm{V} & \mathrm{T} & \mathrm{G} & \mathrm{I} & \mathrm{E} & \mathrm{M} & \mathrm{F} & \mathrm{R} & \mathrm{K} & \mathrm{L} & \mathrm{L} & \mathrm{D} & \mathrm{E} & \mathrm{G} & \mathrm{Q} & \mathrm{A}\end{array}$

1276 CCGGTGAGAACGTCGGTCTGCTGCTTCGCGGCATCAAGCGCGAGGACGTCGAGCGCGGCCAGGTCATCATCAAGC $\begin{array}{llllllllllllllllllllllllll}G & E & N & V & G & L & L & L & R & G & I & K & R & E & D & V & E & R & G & Q & V & I & I & K & P\end{array}$

1351 CGGGCTCGGTCACCCCGCACACCGAGTTCGAGGCCCAGGCCTACATCCTGTCGAAGGACGAGGGTGGCCGTCACA $\begin{array}{lllllllllllllllllllllllllllll}G & S & V & T & P & H & T & E & F & E & A & Q & A & Y & I & L & S & K & D & E & G & G & R & H & T & & & & \end{array}$ CCCCCTTCTTCAACAACTACCGTCCGCAGTTCTACTTCCGTACGACGGACGTGACCGGCGTCGTGACCCTCCCCG $\begin{array}{llllllllllllllllllllllllll}\mathrm{P} & \mathrm{F} & \mathrm{F} & \mathrm{N} & \mathrm{N} & \mathrm{Y} & \mathrm{R} & \mathrm{P} & \mathrm{Q} & \mathrm{F} & \mathrm{Y} & \mathrm{F} & \mathrm{R} & \mathrm{T} & \mathrm{T} & \mathrm{D} & \mathrm{V} & \mathrm{T} & \mathrm{G} & \mathrm{V} & \mathrm{V} & \mathrm{T} & \mathrm{L} & \mathrm{P} & \mathrm{E}\end{array}$

1501 AGGGCACCGAGATGGTCATGCCGGGTGACAACACCGAGATGAAGGTGGAGCTCATCCAGCCCGTCGCCATGGAAG $\begin{array}{lllllllllllllllllllllllllllll}G & T & E & M & V & M & P & G & D & N & T & E & M & K & V & E & L & I & Q & P & V & A & M & E & E\end{array}$

1576 AGGGCCTGAAGTTCGCCATCCGCGAGGGTGGCCGGACCGTGGGCGCCGGCCAGGTCACCAAGATCAACAAGTAAC $\begin{array}{lllllllllllllllllllllllll}G & L & K & F & A & I & R & E & G & G & R & T & V & G & A & G & Q & V & T & K & I & N & K & \star\end{array}$

1651 TCCGCTTGCTTGTCGGTCGACCGACCTGACATGGGCTGATGCCTGAAGGGCCGTACGACTTCGGTCGTACGGGTC 
pASCT1-1 resulted in the identification of an ORF of $1194 \mathrm{bp}$, putatively encoding a 396 amino acid protein (Fig. 1). Sequencing of pBSCT3-1 identified an ORF of $1179 \mathrm{bp}$, corresponding to a protein of 391 amino acids (Fig. 2). The ORF located on pASCT1-1 is very similar to $S$. ramocissimus tufl, and a second ORF is located upstream of it that very much resembles the $S$. ramocissimus fus gene, indicating its probable location in the so-called S12 operon, a location typical for the major tuf gene in all microorganisms [10]. Therefore, the gene was designated tufl. Again on the basis of the very high similarity to its homologue in $S$. ramocissimus, the ORF identified on pBSCT3-1 was designated tuf3.

An amino acid alignment of the tuf-gene products of $S$. coelicolor, $S$. ramocissimus and $E$. coli is shown in Fig. 3. From this alignment it follows that the tuf1 and tuf3 gene products contain the consensus sequences for GTP binding proteins [11] and show a perfect fit with the D-loop motif [12]. From alignments of both the tuf-gene nucleotide sequences and the amino acid sequences deduced thereof, identities were calculated (Table 1). The tuf1 genes of $S$. coelicolor and $S$. ramocissimus are $94 \%$ identical, and the deduced amino acid sequences of their gene products (designated EFTu1) are $96 \%$ identical. The $t u f 3$ genes of these organisms are $87 \%$ identical, their gene products (designated EF-Tu3) sharing $91 \%$ identical amino acids. The low similarity of EF-Tu3 to EF-Tu1, the major EF-Tu in Streptomyces, is underlined by the fact that EF-Tu3

GGTACCGCTCTCGAACGGCCGTTCCATAAAAAACCATTCGACGTGCGACGAAGCGTCGGCGATGATCTGTCTCAT GTTCCGGTACGCCTTCCACCTCGCAGCATCCGCGGTCGCGGATGCCCCGAAGGCTGCCGTCGCCCACTTCACCGC CGCAGTCGACGGCGCCCGAAGCTGACCCTCCCCGGATCGTCCGGCGGACCCCGCAGGGGGAGGGTCGGCCGGCCC CAGGGGTCCCCACCACCGCCCACGAGGCTTCGAGGTACCGCCATGTCCAAGACGGCGTACGTCCGCACCAAACCG $\begin{array}{lllllllllllllll}M & S & K & T & A & Y & V & R & T & K & P\end{array}$

CATCTGAACATCGGCACGATGGGCCATGTCGACCACGGCAAGACCACCCTGACCGCCGCCATCACCAAGGTCCTC $\begin{array}{llllllllllllllllllllllllll}\text { H } & \text { L } & N & \text { I } & G & \text { T } & M & G & \text { H } & \text { V } & \text { D } & \text { H } & G & \text { K } & \text { T } & \text { T } & \text { L } & \text { T } & \text { A } & \text { A } & \text { I } & \text { T } & \text { K } & \text { V } & \text { L }\end{array}$ GCCGAGCGCGGGGCCGGCAGCACCACCCAGTACGTTTCGTTCGACCGCATCGACCGCGCCCCGGAGGAGGCGGCG $\begin{array}{lllllllllllllllllllllllll}A & E & R & G & A & G & S & T & T & Q & Y & V & S & F & D & R & I & D & R & A & P & E & E & A & A\end{array}$ CGCGGCATCACCATCAACATCGCGCACGTCGAGTACGAAACCGACACCCGGCACTACGCCCACGTCGACATGCCC $\begin{array}{lllllllllllllllllllllllllll}R & G & I & T & I & N & I & A & H & V & E & Y & E & T & D & T & R & H & Y & A & H & V & D & M & P\end{array}$ GGCCACGCCGACTACGTCAAGAACATGGTCACCGGCGCCGCCCAGCTCGACGGGGCGATCCTCGTCGTATCCGCG $\begin{array}{lllllllllllllllllllllllll}G & H & A & D & Y & V & K & N & M & V & T & G & A & A & Q & L & D & G & A & I & L & V & V & S & A\end{array}$ CTGGACGGGATCATGCCGCAGACCGCCGAACACGTGCTGCTCGCCCGGCAGGTGGGCGTCGACCACATCGTGGTC $\begin{array}{llllllllllllllllllllllllll}L & D & G & I & M & P & Q & T & A & E & H & V & L & L & A & R & Q & V & G & V & D & H & I & V & V\end{array}$ GCGCTCAACAAGGCCGACGCGGGTGACGAGGAGCTGACCGACCTGGTCGAGCTGGAGGTGCGCGAACTGCTCACC $\begin{array}{lllllllllllllllllllllllll}\text { A } & \text { L } & \text { N } & \text { K } & \text { A } & \text { D } & \text { A } & \text { G } & \text { D } & \text { E } & \text { E } & \text { L } & \text { T } & \text { D } & \text { L } & \text { V } & \text { E } & \text { L } & \text { E } & \text { V } & \text { R } & \text { E } & \text { L } & \text { L } & \text { T }\end{array}$ GCGCACGGCTACGGCGGCGACGCCGTGCCCGTCGTACGGGTCTCGGGGCTGAAGGCCCTGGAGGGCGACCCGCGG $\begin{array}{lllllllllllllllllllllllll}\text { A } & \text { H } & \text { G } & \text { Y } & \text { G } & \text { G } & \text { D } & \text { A } & \text { V } & \text { P } & \text { V } & \text { V } & \text { R } & \text { V } & \text { S } & \text { G } & \text { L } & \text { K } & \text { A } & \text { L } & \text { E } & \text { G } & \text { D } & \text { P } & \text { R }\end{array}$ TGGACGGCCTCCGTCGAGGCGCTGCTCGACGCCGTGGACACGTACGTGCCCATGCCCGAGCGGTACCTGGACGCG $\begin{array}{llllllllllllllllllllllllll}W & T & A & S & V & E & A & L & L & D & A & V & D & T & Y & V & P & M & P & E & R & Y & L & D & A\end{array}$ CCGTTCCTGCTGCCGGTGGAGAACGTGCTCACCATCACCGGCCGCGGCACCGTCGTCACCGGCGCCGTCGAGCCG $\begin{array}{lllllllllllllllllllllllll}P & F & L & L & P & V & E & N & V & L & T & I & T & G & R & G & T & V & V & T & G & A & V & E & P\end{array}$ GGCACCGTGCGCGTCGGCGACCGGGTCGAGGTGCTCGGGGCGTCCGTCGAGACGGTCGTCACCGGCCTGGAGACC $\begin{array}{lllllllllllllllllllllllll}G & T & V & R & V & G & D & R & V & E & V & L & G & A & S & V & E & T & V & V & T & G & L & E & T\end{array}$ TTCGGCAAGCCGATGGAGGAGGCGCAGGCCGGGGACAACGTGGCGCTGCTGCTGCGCGGGGTCGCCCGCGACACG $\begin{array}{lllllllllllllllllllllllllll}\text { F } & G & \text { K } & \text { P } & \text { M } & \text { E } & \text { E } & \text { A } & \text { Q } & \text { A } & \text { G } & \text { D } & \text { N } & \text { V } & \text { A } & \text { L } & \text { L } & \text { L } & \text { R } & \text { G } & \text { V } & \text { A } & \text { R } & \text { D } & \text { T }\end{array}$

26 GTGCGCCGCGGGCAGGTGGTCGCCGCACCCGGCAGCGTCGTCCCCGCGCGGCGTTTCCGGGCCCGGGTGTACGTG $\begin{array}{lllllllllllllllllllllllllll}V & R & R & G & Q & V & V & A & A & P & G & S & V & V & P & A & R & R & F & R & A & R & V & Y & V\end{array}$ CTCTCGGCGCGCGAGGGCGGGCGCTCGACACCGCTCACCACCGGATACCGGCCGCAGTTCTACATCCGCACCGCC

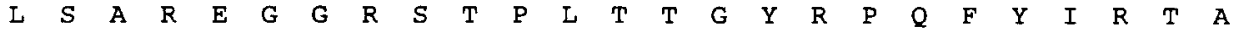
GACGTGGTCGGCGACGTGGACCTCGGCGAGGAGGCCGTCGCCCGGCCCGGGGACACCGTCACCATGACGGTCGAG $\begin{array}{lllllllllllllllllllllllll}\mathrm{D} & \mathrm{V} & \mathrm{V} & \mathrm{G} & \mathrm{D} & \mathrm{V} & \mathrm{D} & \mathrm{L} & \mathrm{G} & \mathrm{E} & \mathrm{E} & \mathbf{A} & \mathrm{V} & \mathrm{A} & \mathrm{R} & \mathbf{P} & \mathrm{G} & \mathrm{D} & \mathrm{T} & \mathrm{V} & \mathrm{T} & \mathbf{M} & \mathbf{T} & \mathrm{V} & \mathrm{E}\end{array}$ CTGGGACGGGACGTGCCGCTGGAGACGGGGCTCGGCTTCGCGATCCGCGAGGGCGGTCGCACCGTGGGGGCGGGG $\begin{array}{llllllllllllllllllllllllll}\text { L } & G & R & D & V & P & \text { L } & E & \text { T } & G & \text { L } & G & \text { F } & \text { A } & \text { I } & \text { R } & \text { E } & G & G & \text { R } & \text { T } & \text { V } & G & \text { A } & G\end{array}$ 26 ACCGTGACCGCGGTGGAGTGAGCGCGCCGGGGTGAGTGGGCCGGGTGAGGGC 1478 $\mathrm{T} \quad \mathrm{V} \quad \mathrm{T}$ A $\mathrm{V}$ E *

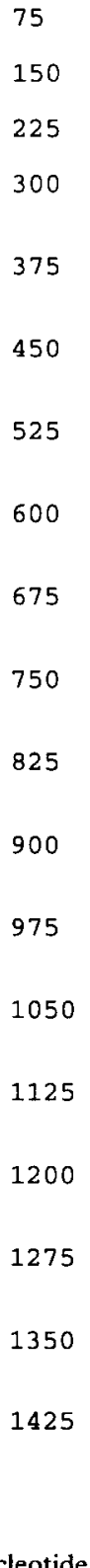

150

00

Fig. 2. Nucleotide sequence of $t u f 3$ and flanking sequences. The deduced amino acids sequence of $t u f 3$ is given below the nucleotide sequence. 
SC $E F-T u I$

Sr $E F-T u 1$

SC $E F-T u 3$

Sr $E F-T u 3$

Sr EF-TU2

EC EF-TUA

Consensus

SC EF-Tu1

Sr EF-Tu1

SC EF-Tu3

Sr EF-Tu3

Sr EF-Tu2

EC EF-TUA

Consensus

SC EF-TU1

Sr EF-TuI

SC EF-TU

ST EF-TU 3

Sr EF-TU2

EC EF-TUA

Consensus

SC EF-TUI

Sr EF-TuI

SC EF-TU3

Sr EF-Tu 3

Sr EF-TU2

EC EF-TUA

Consensus

SC EF-TU1

Sr EF-TuI

SC EF-TU3

Sr EF-TU3

Sr EF-TU2

EC EF-TUA

Consensus

SC EF-TU1

Sr EF-TuI

SC $E F-T u 3$

Sr EF-Tu

Sr EF-Tu2

EC EF-TUA

Consensus

Sc EF-TuI

Sr EF-TUI

SC EF-Tu 3

Sr EF-Tu3

SI EF-TU2

EC EF-TUA

Consensus

SC EF-TuI

Sr EF-TuI

$S C$ EF-Tu 3

Sr EF-Tu 3

Sr EF-Tu2

EC EF-TUA

Consensus
VAKAKFERTK PHVNIGTIGH IDHGKTTLTA AITKVLHDAY PD. INEASAF

\begin{tabular}{|c|c|c|}
\hline MS TAYV & $\mathrm{L}$ & \\
\hline $\begin{array}{r}\text { MS TAYV } \\
Q\end{array}$ & L & 1 \\
\hline
\end{tabular}

AERG AGSTTOYVS

AERG SGT...FVP

$\mathrm{S} E-\mathrm{V} \quad \mathrm{V}$

T AKT GG... A R

$\mathrm{V}-\mathrm{K}-\mathrm{KF}-\mathrm{RTK}$ PHVNIGTIGH -DHGKTILTA AITKVL- - - - - - - F

DQIDKAPEER QRGITISIAH VEYQTEARHY AHVDCPGHAD YIKNMITGAA 99

\begin{tabular}{|c|c|c|c|c|c|c|}
\hline R & $A A$ & $\mathrm{~N}$ & $\mathrm{E} D \mathrm{~T}$ & M & $\mathrm{V}$ & V \\
\hline $\mathrm{R}$ & A A & $\mathrm{N}$ & E DT & $\mathrm{M}$ & V & $\mathrm{V}$ \\
\hline $\mathrm{N}$ & $\mathrm{K} A$ & NTS & $\mathrm{D}$ PT & & V & \\
\hline
\end{tabular}

D-ID-APEE- -RGITI-IAH VEY-T--RHY AHVDCPGHAD Y-KNMITGAA

QMDGAILVVA ATDGPMPQTK EHVLLARQVG VPYIVVALNK ADMVDDEEIL 1.49

$\begin{array}{lllllllll}\mathrm{L} & \text { S } & \text { L } & \text { I } & \text { A } & \text { DH } & \text {. AG } & \text { LT } & 149 \\ \text { L } & \text { S } & \text { L } & \text { I } & \text { A } & \text { DH } & \text {.AG } & \text { LT } & 146\end{array}$

$\begin{array}{llllllll}\text { R I G } & \text { I F } & \text { T } & \text { L } & 149 \\ \end{array}$

QMDGAILWVA ATDGPMPQT - EHVLLARQVG VPYIVVALNK ADMVDDEE--

ELVELEVREL LSEYEFPGDD VPVVKVSALK ALEGDKEWGN SVLELMKAVD 199

$\begin{array}{llllll} & \text { L } & \text { R } & \text { AQ TQ } & \text { D } & 199\end{array}$

$\begin{array}{lllllllll}D & \text { TAHGYG } & \text { A } & \text { R } & \text { G } & \text { PR TA } & \text { EA } & \text { LD } & 199\end{array}$

D D HGYG G A $\quad$ R $G \begin{array}{llllll} & \text { P } & \text { PK TA } & \text { IEA LD } & 196\end{array}$

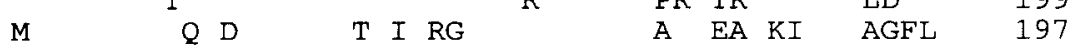

ELVELEVREL LSEY-FPODD -PVVRVSALK ALEGD-WT- SVL-L--AVD

EAIPEPERDV DKPFLMPIED VFTITGRGTV VTGRIERGVL KVNETVDIIG 249

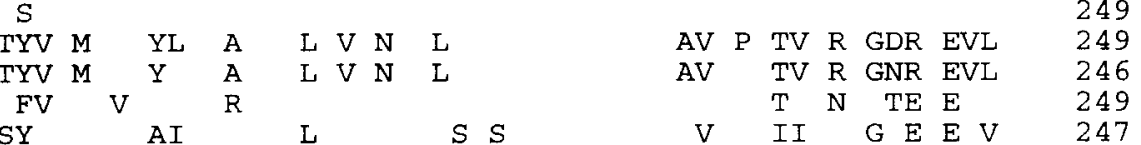

--PEPER-V D-PFL-PIED VFTITGRGTV VTGR-ERG-.- -V---VEI-G

IKTEKTTTTV TGIEMFRKLL DEGQAGENVG LLLRGIKRED VERGQVIIKP 299

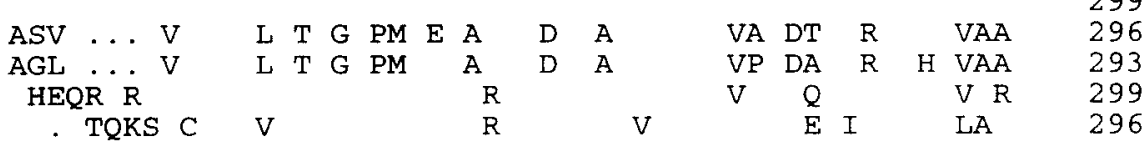

I--E---TWV TE-BMTRKLI DEQQAGENVG LLLRG-KRE- VERGQV--D

GSVTPHTEFE AQAYILSKDE GGRHTPFFNN YRPQFYFRTT DVTGVVTLPE 349

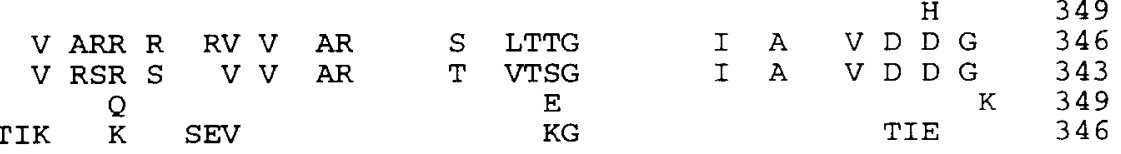

GSV-PHT-FE AO-YTLSKDE GQRHPFH-- YRPOFYTRTT DVTG-V-LPE

GTEMVMPGDN TEMKVELIQP VAMEEGLKFA IREGGRTVGA GQVTKINK 397

$\begin{array}{lllll} & R & V & 397\end{array}$

$\begin{array}{llllllllll}\text { E.AVAR } & \text { T VT } & \text { T } & \text { GRD } & \text { PL } & \text { T } & \text { G } & \text { T } & \text { AVE. } & 392\end{array}$

$\begin{array}{llllllll}\text { V.GVAR ET VS I GRE PL } & \text { P } & \text { G } & \text { T } & \text { ALV. } & 389\end{array}$

$\begin{array}{lllll}A & \mathrm{Q} & \mathrm{I} & \mathrm{R} \mathrm{V} & 397\end{array}$

$\begin{array}{lllllllll}\text { V IK V T } & H & \text { I } & \text { DD } & R & \text { V A VLG } & 394\end{array}$ 
Table 1

Nucleotide sequence identities between the tuf genes of $S$. coelicolor, $S$. ramocissimus and $E$. coli (above the diagonal) and amino acid identities between their deduced gene products (below the diagonal)

\begin{tabular}{lllllll}
\hline & Sc 1 & Sc 3 & Sr 1 & Sr 2 & Sr 3 & Ec A \\
\hline Sc tuf1, EF-Tu1 & & 69 & 94 & 84 & 70 & 71 \\
Sc tuf3, EF-Tu3 & 63 & & 69 & 70 & 87 & 61 \\
Sr tuf1, EF-Tu1 & 96 & 63 & & 85 & 70 & 71 \\
Sr tuf2, EF-Tu2 & 89 & 64 & 88 & & 70 & 69 \\
Sr tuf3, EF-Tu3 & 64 & 91 & 65 & 64 & & 63 \\
Ec tufA, EF-TuA & 75 & 60 & 74 & 72 & 60 &
\end{tabular}

All values are given in percentages. Abbreviations: Sc, S. coelicolor; $\mathrm{Sr}, S$. ramocissimus; and Ec, E. coli. Alignments were done with the program 'Gap' [13].

shows almost as much amino acid identity with $E$. coli EF-Tu as with $S$. coelicolor EF-Tu1.

A surprising observation is that the nucleotide sequences of tufl and tuf 3 are more homologous to each other than the deduced amino acid sequences (69\% versus $63 \%$ ), which is also observed for the $S$. ramocissimus tuf1 and tuf3 genes and their gene products (Table 1). This is due to nucleotide substitutions in the tuf 3 gene at the first and second codon position rather than the third ('wobble') position, so that many nucleotide substitutions lead to amino acid substitutions. Still, amino acids in the GTP-binding domains are conserved, suggesting that the tuf 3 gene product also belongs to the family of GTP-binding proteins.

Since it has been demonstrated for $S$. ramocissimus EF-Tu1 that it functions as an elongation factor [4] we assume on the basis of the very high similarity of the proteins that such is also the case for $S$. coelicolor EF-Tu1 and no further investigation to address this point has been performed. The role of EF-Tu3 is unclear and is presently being investigated in our laboratory.

\section{References}

[1] An, G. and Friesen, J.D. (1980) Gene 12, 33-39.

[2] Yokota, T., Sugisaki, H., Takanami, M. and Kaziro, Y. (1980) Gene 12, 25-31.

[3] Van der Meide, P.H., Vijgenboom, E., Talens, A. and Bosch, L. (1983) Eur. J. Biochem. 130, 397-407.

[4] Vijgenboom, E., Woudt, L.P., Heinstra, P.W.H., Rietveld, K., Van Haarlem, J., Van Wezel, G.P., Shochat, S. and Bosch, L. (1994) Microbiology 140, 983-998.

[5] Sela, S., Yogev, D., Razin, S. and Bercovier, H. (1989) J. Bacteriol. 171, 581-584.

[6] Filer, D. and Furano, A.V. (1981) J. Bacteriol. 148, 1006-1011.

[7] Van Wezel, G.P (1994) PhD thesis, Leiden University, Leiden, Netherlands.

[8] Twigg, A.J. and Sherratt, D. (1980) Nature 283, 216-218.

[9] Covarrubias, L. and Bolivar, F. (1982) Gene 17, 79-89.

[10] Lindahl, L. and Zengel, J.M. (1986) Annu. Rev. Genet. 20, 297-326.

[11] Dever, T.E., Glynias, M.J. and Merrick, W.C. (1987) Proc. Natl. Acad. Sci. USA 84, 1814-1818.

[12] Saraste, M., Sibbald, P.R. and Wittinghofer, A. (1990) Trends in Biochem. Sci. 15, 430-434.

[13] Devereux, J., Haeberli, P. and Smithies, O. (1984) Nucleic Acids Res. 12, 387-395.

Fig. 3. Amino acid alignment of Streptomyces EF-Tus and E. coli EF-TuA. Abbreviations: Ec, E. coli; Sc, S. coelicolor; Sr, S. ramocissimus. Numbers at the right of the figure refer to the amino acid positions. A consensus (grey shaded) is given when the amino acid alignment shows more than three identical amino acids. The amino acids (of Sc EF-Tu1) that constitute the GTP binding consensus sequence are shown in bold face. 